\title{
Role of structural fluctuations in the insertion into complex host matrices
}

\author{
E.V. Vakarin and J.P. Badiali \\ LECA ENSCP-UPMC, 11 rue P. et M. Curie, 75231 Cedex 05, Paris, France
}

\begin{abstract}
A coupling of structural and thermodynamic fluctuations in the course of various-type insertion processes is investigated within a combination of Gibbsian statistics and the information theory approach. It is shown that the coupling makes it possible to restore (at least partially) the information, inaccessible from experimental tests. This enables one to make physically reasonable predictions under limited information on the system.
\end{abstract}

\section{INTRODUCTION}

Intercalation processes find their application in many technologically important domains, such as the design of hydrogen-storage systems [1], rechargeable high-energy batteries, electrochromic devices, (see Ref. [2] for a review), electroactive polymers, and superconductors [3]. Microscopically the insertion is a complex process involving many effects (e.g. the charge transfer, elastic response of the matrix, the permselectivity, etc). Even well-characterized materials, like crystalline or layered compounds, exhibit rather complicated elastic properties, involving restructuring, staging and random distortion of the galleries [4]. For amorphous (or porous) matrices [5] the situation is complicated by the host heterogeneity, that may change in the course of intercalation. For instance, structural heterogeneity of electroactive polymers changes because of the polymer swelling. Complex geometry and poorly characterized energetics [6] make it practically impossible to construct a mechanical model. Therefore, statistical mechanical investigations in this domain face difficulties, induced by the system complexity. In this situation it seems reasonable to develop a new theoretical scheme, that would be capable of making reasonable predictions under restricted information.

For this purpose we combine the standard statistical mechanics and the information theory approach [7]. The latter has been introduced as an alternative (with respect to the standard statistical mechanics) tool for the statistical description of many-body systems. Such an approach has been successfully applied to a description of nonequilibrium steady states and the self-organization [8] in various complex (physical, chemical, biological, etc.) systems. One of the main advantages of the information theory is its capability of describing the equilibrium and nonequilibrium states in the framework of a unified scheme, resembling the usual Gibbs statistics, provided that a suitable information (entropy) measure is chosen. On the other hand, the approach does not rely upon microscopic details. This allows one to study the systems deviating from the standard equilibrium conditions. Quenched systems, like fluids adsorbed in porous media [9], spin glasses $[10,11]$ or liquid glassformers [12] could serve as relevant examples.

In this paper we consider complex systems in which the information at a microscopic level is not sufficient for making a link between the system mechanics (Hamiltonian) and its statistics (probability distribution). Then the we can split the system into subsystems with different levels of description. One is a dynamic subsystem, which evolves according to a Hamiltonian, containing unknown parameters. The latter reflect a coupling to a stochastic subsystem which is governed by a probability distribution. This differs from the usual quenching in, at least, two aspects. The dynamic subsystem can influence the stochastic one and their coupling determines both the thermodynamic behavior and the shape of the probability distribution. For instance, by the analogy with the intercalation system [13-15], we can expect a change of the matrix volume upon the fluid absorption. Secondly, one necessarily deals with two entropic impacts: the thermodynamic entropy (due to the dynamic subsystems) and the information entropy (due to the probability distribution). Constructing from these two a suitable entropy measure, we investigate the strain distribution and the guest thermodynamics in homogeneous and heterogeneous matrices.

\section{MAXIMUM ENTROPY ESTIMATION}

Let us assume that the states of a system are labelled by a continuous variable $x$, with $p(x)$ being the probability of a state $x$. Nevertheless, because of the system complexity, the probabilities are not known a priori. The only available information is on a constrained quantity

$$
Q=\int d x p(x) q(x)
$$

which is the expectation value of a physical observable (e.g. energy, heat flux, etc.). In addition the probability distribution is assumed to be normalized

$$
\int d x p(x)=1
$$

Given this information can we estimate another relevant quantity $G=\int d x p(x) g(x)$ ? For this pur- 
pose one has to restore the probability distribution $p(x)$.

The basic role in this approach is played by the entropy $S$ that gives a measure of missing information [7] concerning the system state. If we have the entropy as a functional of the probability distribution $S=S[p(x)]$, then, according to the information theory approach $[7,8]$, the probability can be estimated through the entropy maximization under constraints (1),(2). If we take the entropy (or the information) measure in the Shannon form

$$
S=-\int d x p(x) \ln p(x)
$$

then such a variation procedure gives

$$
p(x)=\frac{e^{-\gamma q(x)}}{Z} ; \quad Z=\int d x e^{-\gamma q(x)}
$$

where $\gamma$ is a Lagrange multiplier which has to be determined from the constraint (1). For instance, if $Q$ is associated with the equilibrium internal energy, then $p(x)$ becomes the conventional Gibbs distribution, with $\gamma=1 /(k T)$.

\section{COMPLEX SYSTEMS}

As is discussed above, by complexity we mean a situation when the available microscopic information on a system is not sufficient for its description in terms of a Hamiltonian. Thus, only one subsystem, say $\{s\}$, is governed by a Hamiltonian $H[\sigma, s]$, while the rest (the surrounding) is specified by a set of relevant parameters $\{\sigma\}$ which appear with a probability distribution $P(\sigma)$. Therefore, we have a coupling of a dynamic system to a stochastic one, which however influence each other. This means that $P(\sigma)$ is unknown, the only available information concerns with an observable quantity

$$
E=\int d \sigma P(\sigma) E(\sigma)
$$

that gives, for instance, a characteristic energy scale for the surrounding. In the case of spin glasses the counterparts of $\{s\}$ and $\{\sigma\}$ systems are the spin variables and the random fields (or exchange constants), respectively. For porous media, $\{\sigma\}$ can be identified with the matrix (e.g. pore size) and $\{s\}$ - with the adsorbate degrees of freedom.

For a given configuration $\{\sigma\}$ one can calculate the partition function of the dynamic subsystem

$$
Z(\sigma)=\int(d s) e^{-\beta H[\sigma, s]}
$$

where $\beta=1 /(k T)$ is the inverse temperature. Then all the thermodynamic characteristics are known. For instance, the free energy

$$
F(\sigma)=-\frac{1}{\beta} \ln Z(\sigma)
$$

and the internal energy

$$
U(\sigma)=-\frac{d}{d \beta} \ln Z(\sigma)
$$

determine the thermodynamic entropy

$$
S_{T}(\sigma)=\beta[U(\sigma)-F(\sigma)]
$$

Then the relevant thermodynamic quantities can be obtained by averaging over all realizations of $\sigma$ . For instance, the average free energy is given by

$$
F=\langle F(\sigma)\rangle=\int(d \sigma) P(\sigma) F(\sigma)
$$

Note that, in contrast to the conventional equilibrium, now we have two entropies - the thermodynamic entropy

$$
S_{T}=\int(d \sigma) P(\sigma) S_{T}(\sigma)
$$

and the one related to the information on the probability distribution

$$
S_{I}=-\int(d \sigma) P(\sigma) \ln P(\sigma)
$$

For a given distribution $P(\sigma)$ the value of $S_{T}$ is the average thermodynamic entropy of the coupling $\{s\}-\{\sigma\}$. If, however, the distribution is not known, then $S_{T}$ can be viewed as an additional entropy measure for inferring $P(\sigma)$ through the variation procedure.

In the case when the surrounding affects the dynamic subsystem, but not vice versa (pure quenching) $P(\sigma)$ can be determined through the $S_{I}$ maximization under the constraint (5) and the normalization condition. This gives

$$
P(\sigma)=\frac{e^{-\gamma E(\sigma)}}{Z_{0}} \quad Z_{0}=\int d \sigma e^{-\gamma E(\sigma)}
$$

where $E(\sigma)$ can be estimated through model or even scaling arguments. For instance, $E(\sigma)=a \sigma^{2}$ is the energy to create a field of the magnitude $\sigma$. As is discussed above, the Lagrange multiplier $\gamma$ should be determined from the condition (5). Once the probability distribution is determined, the thermodynamic quantities can be calculated straightforwardly.

Such a decoupling of the two subsystems is however an idealization, which could be more or less acceptable depending on a concrete situation. In general, the surrounding should respond to the evolution of the dynamic subsystem. For instance, the porous materials could change the volume 
upon accommodation of the fluid, like in the case of intercalation systems [13-15]. Also, it has been demonstrated [16] that the effect of the quenching on the thermodynamics of $\mathrm{HCl}$-ice interfaces weakens with increasing ice film thickness.

If the subsystems do influence each other, then the probability distribution must be evaluated starting from the total entropy $\Sigma\left(S_{T}, S_{I}\right)$, that describes the uncertainty concerning the state of the overall system. Obviously, $\Sigma\left(0, S_{I}\right)=S_{I}$ and $\Sigma\left(S_{T}, 0\right)=S_{T}$ when there is no loss of information on one of the subsystems. The main problem is to construct $\Sigma\left(S_{T}, S_{I}\right)$ for nonvanishing $S_{T}$ and $S_{I}$. For instance, we can formally expand around the perfectly ordered state $S_{I}=0, S_{T}=0$

$$
\Sigma\left(S_{T}, S_{I}\right)=\Sigma(0,0)+\kappa S_{T}+\lambda S_{I}+\delta S_{T} S_{I}+\ldots
$$

As a first approximation the total entropy $\Sigma\left(S_{T}, S_{I}\right)$ can be estimated as a quasi-additive combination (we drop $\Sigma(0,0)$ as an irrelevant constant)

$$
\Sigma\left(S_{T}, S_{I}\right)=S_{I}+\kappa S_{T}
$$

where $\kappa$ is a parameter reflecting the coupling between the dynamic and the stochastic subsystems. Then the distribution function can be inferred by maximizing (15) under the constraint (5). This leads to

$P(\sigma)=\frac{e^{-\gamma^{\prime} E(\sigma)+\kappa S_{T}(\sigma)}}{Z} \quad Z=\int d \sigma e^{-\gamma^{\prime} E(\sigma)+\kappa S_{T}(\sigma)}$

where the Lagrange multiplier $\gamma^{\prime}$ should be determined from the constraint (5). It is known [17] that the entropy $S_{T}(\sigma)$ determines the probability of thermodynamic fluctuations for a given value of $\sigma$ according to

$$
P_{\varphi}(\sigma)=\frac{e^{\kappa S_{T}(\sigma)}}{\int d \sigma e^{\kappa S_{T}(\sigma)}}
$$

It should be emphasized that $P_{\varphi}(\sigma)$ does not describe the fluctuation of $\sigma$, but the fluctuation of thermodynamic variables related to $\{s\}$-subsystem (e.g. temperature, density, etc) for a given value of $\sigma$. Then the resulting distribution (16) can be represented as

$$
P(\sigma)=P_{\varphi}(\sigma) \frac{e^{-\gamma^{\prime} E(\sigma)}}{\left\langle e^{-\gamma^{\prime} E(\sigma)}\right\rangle_{\varphi}}
$$

where the average $\langle\ldots\rangle_{\varphi}$ is taken over the thermodynamic fluctuations of the dynamic subsystem. We thus see that the statistics involves two ingredients: the "energetic", $E(\sigma)$ and the entropic $S_{T}(\sigma)$ or $P_{\varphi}(\sigma)$. On the other hand, all thermodynamic quantities (like $S_{T}=\left\langle S_{T}(\sigma)\right\rangle$ ) are also affected by these impacts.

\section{STRAIN DISTRIBUTION IN THE COURSE OF INSERTION}

In this section we analyze the interplay of these two impacts considering various aspects related to insertion systems. In this case the guest plays the role of $s$-subsystem, whose coupling to the host matrix is given by the matrix strain $\varepsilon$ which is a counterpart of $\sigma$-subsystem. We are interested in determining the strain distribution $P(\varepsilon)$ and the guest thermodynamics $S_{T}$, taking into account the host-guest coupling $S_{T}(\varepsilon)$.

\section{A. Homogeneous matrix}

As an illustration of our approach we consider the simplest example of guest insertion into a "homogeneous" host matrix. "Homogeneous" means that the matrix properties (the structure, the binding site distribution, etc.) are similar for different matrix domains (e.g. graphite galleries). It is wellknown that the matrix becomes strained with increasing guest concentration $x$. For a given $x$ the actual strain is a sum of the domain strains $\sum_{i} \varepsilon_{i}$. For homogeneous matrices we may expect that all the domains are equivalent, such that $\sum_{i} \varepsilon_{i}=n \varepsilon$, where $n$ is the number of domains.

Therefore, the host-guest coupling leads to a strain distribution $P(\varepsilon)$ and affects the guest thermodynamics. The strain-dependent guest entropy $S_{T}(\varepsilon)$ can be expanded around $\varepsilon=0$

$$
S_{T}(\varepsilon)=S_{T}(0)+\alpha n \varepsilon+\ldots
$$

Here $S_{T}(0)$ is the guest entropy in the case when the matrix is not strained. This corresponds to the lattice gas model [2] describing the guest adsorption on a rigid lattice. Note that all the quantities above $\left(S_{T}(0), \alpha, \varepsilon\right)$ are concentration dependent. The strain distribution $P(\varepsilon)$ can be determined by maximizing $S_{I}+\kappa S_{T}$ under the constraint that the average strain

$$
E=n\langle\varepsilon\rangle=n \int d \varepsilon P(\varepsilon) \varepsilon
$$

is known (e.g. measured experimentally). It can be estimated as the sample volume dilatation (or $c$-axis expansion for layered compounds).

According to eq. (18) we arrive at

$$
P(\varepsilon)=\frac{e^{-\left(\gamma^{\prime}-\kappa \alpha\right) \varepsilon}}{\int d \varepsilon e^{-\left(\gamma^{\prime}-\kappa \alpha\right) \varepsilon}}
$$

Determining $\gamma^{\prime}$ from the constraint (20) we obtain 


$$
P(\varepsilon)=\frac{e^{-\varepsilon / E}}{\int d \varepsilon e^{-\varepsilon / E}}
$$

It should be noted that exactly the same distribution would be recovered if only $S_{I}$ is used as the entropy measure. This is a consequence of what we called homogeneity, that is the average $E$ is a good estimation of the actual strain $\varepsilon$, which almost does not fluctuate. Then the experimentally available information (20) is relevant to the thermodynamic functions of interest. In other words, if the experimental data allow one to recover an "exhaustive" picture of the system, then any refinement of the entropy measure (like introducing $S_{I}+\kappa S_{T}$ instead of $S_{I}$ ) gives no new information.

Based on the probability distribution the guest thermodynamics can be predicted using the experimental data on the concentration dependence of the strain $E=E(x)$

$$
S_{T}=S_{T}(0)+\alpha E
$$

Having determined $S_{T}$, we can recover all the thermodynamic functions in the standard way. The free energy is also linear in the strain (for simplicity we take the same coefficient $\alpha): F=F(0)+\alpha E$. Based on this we obtain the guest chemical potential as

$$
\mu=\mu(0)+\alpha \frac{d E}{d x}+E \frac{d \alpha}{d x}
$$

This results coincides with the one obtained in our earlier studies $[14,15]$ combining the lattice gas model with the continuum elasticity theory. The latter implies that the stress can be defined as $\alpha=d F / d E$. If the stress $\alpha$ is composition independent, then from (24) we recover the well-known result of Larche and Cahn [18].

\section{B. Heterogeneous matrix}

In reality the host matrices are not homogeneous. Even for materials which are conventionally regarded as well-characterized, the heterogeneity is practically unavoidable in the experimental conditions (e.g. because of defects, domain structures, etc). On the other hand the amorphous or porous matrices are essentially heterogeneous and their properties are known only statistically. In this case it is difficult to "invent" a simple experimental test (like (20)), giving an exhaustive description. We assume that the insertion of guest species into a heterogeneous matrix results in a strain distribution for various matrix domains. For simplicity we do not consider spatial strain correlations. This means that the domain strains $\varepsilon_{i}$ are randomly distributed according to $P\left(\left\{\varepsilon_{i}\right\}\right)=\prod_{i} P\left(\varepsilon_{i}\right)$ independently of the domain position $i$.
From the experimental point of view (e.g. measuring the matrix strain $E$ or from another suitable test) we know only the range in which the individual $\varepsilon_{i}$ can vary. The range of $\varepsilon_{i}$ variation should correlate with the average $E$. For instance, if $E=0$, then $\varepsilon_{i}$ are symmetrically distributed around 0 . For concreteness we choose: $0<\varepsilon_{i}<g_{i}(E)$. Then, maximizing $S_{I}$ under the normalization condition for $P\left(\varepsilon_{i}\right)$, we obtain the following step-wise distribution

$$
P_{0}\left(\varepsilon_{i}\right)=\frac{H\left(\varepsilon_{i}\right) H\left(g_{i}-\varepsilon_{i}\right)}{g_{i}}
$$

where $H(x)$ is the Heaviside step function. The distribution $P_{0}\left(\varepsilon_{i}\right)$ is the one we can recover using the experimentally available information.

The guest thermodynamics can be predicted as before

$$
\begin{aligned}
& S_{T}=S_{T}(0)+\sum_{i} \alpha_{i}\left\langle\varepsilon_{i}\right\rangle_{0} \\
& \mu=\mu(0)+\sum_{i} \alpha_{i} \frac{d}{d x}\left\langle\varepsilon_{i}\right\rangle_{0}
\end{aligned}
$$

where $\left\langle\varepsilon_{i}\right\rangle_{0}=g_{i} / 2$ and $\alpha_{i}$ is assumed to be composition independent. Note that the results above are based on the estimation of the internal strains $\left\langle\varepsilon_{i}\right\rangle_{0}$, using the experimentally available information on the "external" (observable from outside) strain $E$. As a simple approximation we may suppose $g_{i}(E)=f\left(S_{i}\right) E / n$, that is, each $\left\langle\varepsilon_{i}\right\rangle_{0}$ contributes to the observed strain $E$ proportionally to the domain size $S_{i}$, with $\sum_{i}\left\langle\varepsilon_{i}\right\rangle_{0}=E$.

These results can be refined if we explicitly take into the host-guest coupling and determine $P\left(\varepsilon_{i}\right)$ using $S_{I}+\kappa S_{T}$. This leads to the distribution

$$
P\left(\varepsilon_{i}\right)=\frac{e^{\kappa \alpha_{i} \varepsilon_{i}}}{\int_{0}^{g_{i}} d \varepsilon_{i} e^{\kappa \alpha_{i} \varepsilon_{i}}}
$$

which is more informative in the sense that now not all values of $\varepsilon_{i}$ are equally probable. The average

$$
\left\langle\varepsilon_{i}\right\rangle=\frac{1+e^{\kappa \alpha_{i} g_{i}}\left(\kappa \alpha_{i} g_{i}-1\right)}{\kappa \alpha_{i}\left(e^{\kappa \alpha_{i} g_{i}}-1\right)}
$$

is different from $\left\langle\varepsilon_{i}\right\rangle_{0}$ obtained above. It is instructive to analyze the origin of this difference. For this purpose we expand $\left\langle\varepsilon_{i}\right\rangle$ in terms of the coupling $\kappa$

$$
\left\langle\varepsilon_{i}\right\rangle=\left\langle\varepsilon_{i}\right\rangle_{0}+\alpha_{i} \kappa \Delta_{i}^{0}+\ldots
$$

where

$$
\Delta_{i}^{0}=\left[\left\langle\varepsilon_{i}^{2}\right\rangle_{0}-\left\langle\varepsilon_{i}\right\rangle_{0}^{2}\right]=g_{i}^{2} / 12
$$

describes the strain fluctuations. The latter are related to the domain size fluctuations and thus 
can be called the structural fluctuations. In the case of homogeneous matrices all the domains are equivalent, then the structural fluctuations vanish $\Delta_{i}^{0}=0$ and $\left\langle\varepsilon_{i}\right\rangle=\left\langle\varepsilon_{i}\right\rangle_{0}$. Therefore, for heterogeneous matrices our estimation of the strain statistics becomes more sensitive to structural fluctuations if we change the entropy measure. This reflects the fact that for heterogeneous matrices the actual strain $\bar{E}$ is different from the one $(E)$ measured as the sample dilatation. Their difference

$$
\bar{E}-E=\sum_{i}\left[\left\langle\varepsilon_{i}\right\rangle-\left\langle\varepsilon_{i}\right\rangle_{0}\right]=\sum_{i} \alpha_{i} \kappa \Delta_{i}^{0}
$$

takes into account the internal deformations which are not detectable experimentally. This result is quite general, it does not rely upon the simple form for $P_{0}\left(\varepsilon_{i}\right)$ and $P\left(\varepsilon_{i}\right)$ discussed here as examples. The only essential fact is that these two are different (compare (13) and (18)). The difference has its physical origin in the mutual coupling of the guest thermodynamics and the host structure. The coupling makes it possible to "restore" (at least partially) the information inaccessible from experimental tests.

Based on the refined distribution (28) we can calculate the guest thermodynamics.

$$
S_{T}=S_{T}(0)+\sum_{i} \alpha_{i}\left\langle\varepsilon_{i}\right\rangle_{0}+\kappa \sum_{i} \alpha_{i}^{2} \Delta_{i}^{0}+\ldots
$$

Considering $S_{T}$ as a measure of the guest thermodynamic fluctuations (see above), we conclude that the latter are coupled to the host structural fluctuations. This reflects the fact that the matrix response to the guest insertion is not only due to the host nature but also involves the host- guest coupling. Comparing the chemical potential

$$
\mu=\mu(0)+\sum_{i} \alpha_{i} \frac{d}{d x}\left\langle\varepsilon_{i}\right\rangle_{0}+\kappa \sum_{i} \alpha_{i}^{2} \frac{d}{d x} \Delta_{i}^{0}
$$

with (27), obtained using the experimental information, we see that (33) gives a more precise estimation, but also contains essential physics. In particular, if $\sum_{i}\left\langle\varepsilon_{i}\right\rangle_{0} \rightarrow 0$, then from (27) one would conclude that the insertion is almost topotactic and that the internal host distortion is irrelevant. In contrast, eq. (33) implies that although the matrix is not strained on average, the expanded and contracted domains do contribute to the guest energetics. This is observed experimentally [5] on various disordered host matrices, which exhibit negligible volume expansion in comparison to wellstructured materials. Nevertheless, the electrochemical response of disordered insertion systems is remarkably different. This can be understood in terms of two effects. First is the configurational transitions [14] described by $\mu(0)$. Structural disorder induces a broad distribution of binding energies on different matrix sites. This leads to quite steep insertion isotherms with multiple short plateaus. A simple model capable of recovering this effect can be found in ref. [19].

The second effect is due to the structural transitions, which are supplemented by structural fluctuations (see eq. (33)). In order to analyze this contribution within our simple model, let us recall that $\left\langle\varepsilon_{i}\right\rangle_{0}=g_{i}(E(x)) / 2$ and $\Delta_{i}^{0}=g_{i}^{2}(E(x)) / 12$. Assuming the simplest non-trivial concentration dependence $g_{i}(E(x))=b_{i} x$, we obtain from (33):

$$
\mu=\mu(0)+\delta \mu+K x
$$

where $\delta \mu=\sum_{i} b_{i} \alpha_{i} / 2$ induces a shift of the isotherm in comparison to the strain free state, and $K=\kappa \sum_{i} \alpha_{i}^{2} b_{i}^{2} / 6$ plays the role of additional repulsive $(K>0)$ interaction. Since the chemical diffusion coefficient is proportional to $d \mu / d x$, it is clear that the structural fluctuations tend to increase diffusivity in comparison to the strain free state. This seems to be a general tendency for disordered matrices, as it follows from experimental studies $[5,20]$.

\section{CONCLUSION}

The coupling of structural and thermodynamic fluctuations in the course of various-type insertion processes is investigated within a combination of Gibbsian statistics and the information theory approach. The main focus is on complex (heterogeneous) insertion systems, where the microscopic information is not sufficient for making a link between the system mechanics (Hamiltonian) and its statistics (probability distribution). Then the system can be viewed as an entropic coupling of a dynamic and a stochastic subsystem. It is shown that the coupling makes it possible to restore (at least partially) the information, inaccessible from experimental tests. This enables one to make physically reasonable predictions under limited information on the system.

It should be emphasized that our results are also applicable to matrices which are initially homogeneous but become heterogeneous in the course of insertion. For instance, this takes place in $L i$ graphite intercalation compounds, which exhibit staging phenomena. In this case the occupied and empty graphite galleries are not equivalent. Also, if a gallery is partially occupied, then one deals with distorted domains [4]. In this case the strain fluctuations as well as their spatial correlation should be taken into account. In a simple approximation this physics is reflected by our result (see eq. (33)). Our approach is also capable of describing the swelling of electroactive polymers in the course of intercalation. We plan to address these issues in a future study. 
[1] L. Schlapbach, A. Zuttel, Nature 414, 353 (2001)

[2] W. R. McKinnon, R. R. Haering, in Modern aspects of Electrochemistry, edited by R. E. White, J. O' M. Bockris, and B. E. Conway (Plenum, NY, 1983), No 15, p.235

[3] J. S. Slusky et al, Nature 410, 343 (2001)

[4] M. F. Thorpe, W. Jin, S. D. Mahanti, Phys. Rev. B 40, 10294 (1989)

[5] C. M. Julien, Materials Sci. and Engineering R 40, 47 (2003)

[6] P. Thibault, J. J. Prejean, L. Puech, Phys. Rev. B 52, 17491 (1995)

[7] E. T. Jaynes, Phys. Rev. 106, 620 (1957)

[8] H. Haken, Synergetics. An Introduction. Nonequilibrium Phase Transitions and Self-Organization in Physics, Chemistry, and Biology (Springer, Berlin, 1983)

[9] J. A. Given, J. Chem. Phys. 102, 2934 (1995)
[10] D. Sherrington, S. Kirkpatrick, Phys. Rev. Lett., 35 (1975) 1792

[11] M. Mezard, G. Parisi, M. Virasoro, Spin Glass Theory and Beyond (Singapore, World Scientific, 1987)

[12] A. Z. Patashinski, M. A. Ratner, Physica A 314, 526 (2002)

[13] E. V. Vakarin, J.P. Badiali, M. D. Levi, D. Aurbach, Phys. Rev. B, 63, 014304 (2001)

[14] E. V. Vakarin, J. P. Badiali, J. Phys. Chem. B 106, 7721 (2002)

[15] E. V. Vakarin, J. P. Badiali, arXiv: condmat/0306559 (2003), Phys. Rev. B (in press)

[16] E. V. Vakarin, J. P. Badiali, Surf. Sci. 513, 431 (2002)

[17] G. Ruppeiner, Rev. Mod. Phys.,67 (1995) 605

[18] F. C. Larche, J. W. Cahn, Acta Metall. 33, 331 (1985)

[19] E. V. Vakarin, M. F. Holovko, Chem. Phys. Lett. 349, 13 (2001)

[20] Y. M. Choi, S. I. Pyun, Solid State Ionics 99, 173 (1997) 